\title{
Caracterização físico-química de vinhos da cv. Viognier com adição de chips de carvalho
}

\author{
Physico-chemical characterization of wines from cv. Viognier with addition of \\ oak chips
}

\author{
DA SILVA, Marjorie Isabela Rodrigues. Discente do Curso Superior de Tecnologia \\ em Viticultura e Enologia \\ IF Sertão-PE-Campus Petrolina Zona Rural. Rodovia BR 325, Km 22, Projeto Senador Nilo Coelho -N4- Pe- \\ trolinaPE- Brasil- CEP: 56300-00/ Telefone (87) 2101-8050/ E-mail: marjorie.isabela@hotmail.com
}

NOGUEIRA, Elis Tatiane da Silva. Esp. em processamento de frutas e hortaliças IF Sertão-PE-Campus Petrolina Zona Rural. Rodovia BR 325, Km 22, Projeto Senador Nilo Coelho -N4- PetrolinaPE- Brasil- CEP: 56300-00/ Telefone (87) 2101-8050/ E-mail: elis.nogueira@ifsertao-pe.edu.br

SANTOS, Renata Gomes de Barros. Esp. em agronegócio

IF Sertão-PE-Campus Petrolina Zona Rural. Rodovia BR 325, Km 22, Projeto Senador Nilo Coelho -N4- PetrolinaPE- Brasil- CEP: 56300-00/ Telefone (87) 2101-8050/ E-mail: renata.gomes@ifsertao-pe.edu.br

CARVALHO, Erika Samantha Santos de. Mestre em ciências de alimentos

IF Sertão-PE-Campus Petrolina Zona Rural. Rodovia BR 325, Km 22, Projeto Senador Nilo Coelho -N4- PetrolinaPE- Brasil- CEP: 56300-00/ Telefone (87) 2101-8050/ E-mail: erikasamantha2@gmail.com

\begin{abstract}
RESUMO
O objetivo do trabalho é avaliar o efeito da utilização de chips de carvalho francês na composição físico-química em vinhos brancos. 0 experimento foi realizado na Escola do Vinho, IF SERTẤO- PE. As microvinificações foram realizadas em garrafões de 20 litros, sendo 4 tratamentos (T0: sem adição de chip; T1: com 2 g.L-1 de chip; T2: com 3 g. L $^{-1}$ de chip; T3: com $4 \mathrm{~g} . \mathrm{L}^{-1}$ de chip), com três repetições cada, sendo cada unidade amostral uma garrafa. Os vinhos foram submetidos as análises clássicas (densidade, $\mathrm{pH}$, acidez total titulável, extrato seco total e reduzido, acidez volátil, açúcares residuais, teor alcoólico e dióxido de enxofre $\left(\mathrm{SO}_{2}\right)$ livre e total e análises de cor por espectrofotometria. 0 trabalho apresentado indicou que o uso de chips de carvalho francês de média tostagem não alterou as características do vinho, atendendo aos requisitos propostos pela legislação brasileira.
\end{abstract}

Palavras-Chave: Submédio Vale do São Francisco, Vinho tranquilo, Fragmentos de carvalho.

\begin{abstract}
The objective of the work is to evaluate the effect of the use of French oak chips on the physical-chemical composition of white wines. The experiment was carried out at Escola do Vinho, IF SERTÃO-PE. Microvinifications were carried out in 20-liter bottles, with 4 treatments (T0: no chip added; T1: with 2 gL-1 chip; T2: with 3 gL-1 chip; T3: with 4 gL-1 chip ), with three repetitions each, each sample unit being a bottle. The wines were subjected to classical analyzes (density, $\mathrm{pH}$, total titratable acidity, total and reduced dry extract, volatile acidity, residual sugars, alcohol content and free and total sulfur dioxide ( $\mathrm{SO2}$ ) and color analysis by spectrophotometry. The work presented indicated that the use of medium toasting French oak chips did not alter the characteristics of the wine, meeting the requirements proposed by Brazilian legislation.
\end{abstract}

Key words: Sub Valley San Francisco, Quiet, medium toast. 


\section{Introdução}

O Brasil é responsável por produzir 300 milhões de litros de vinhos, assumindo a $15^{\circ}$ posição no ranking de maiores produtores da bebida (O.I.V 2018). O Vale do Submédio São Francisco detém a produção de 8 milhões de litros de vinho (LOPES et al, 2008), divididos em brancos, tintos, róses e espumantes. As condições climáticas da região, com uma temperatura média anual de $26^{\circ} \mathrm{C}$, alta luminosidade e água disponível para irrigação a partir do Rio São Francisco, propiciam ciclos vegetativos constantes, intercalados, com colheitas nos vários meses do ano (BIROLO; ZANELLA, 2017). Estas condições possibilitam que ocorra o planejamento de todo processo de elaboração do vinho, desde a data de colheita ao engarrafamento (PEREIRA, 2010).

A uva destinada para elaboração do vinho branco tranquilo deve apresentar estrutura ácida considerável, pois a acidez é responsável pelas características de frescor do produto (GUERRA et al., 2009). A uva, em sua composição, apresenta constituintes primordiais para a qualidade do vinho produzido, sendo eles, açúcares indicando a potencial alcoólico da bebida, ácidos orgânicos responsáveis pela qualidade organoléptica, aminoácidos e precursores de aromas (SANTOS et al., 2009).

A variedade Viognier é uma uva vinífera branca originária da França, é conhecida por dar origem a vinhos com aromas florais, além de serem complexos, poderosos e de alta qualidade (JOHNSON; ROBINSON, 2007). A cultivar Viognier apresenta seus cachos e bagas compactados, é sensível ao vento, normalmente conduzida em poda longa e alta densidade de plantio, apresentando brotações precoces (TOGORES, 2011). Esta variedade origina vinhos brancos bem estruturados e secos, de cor amarelo forte, com aromas complexos de damasco, graviola e pêssego (SILVA, 2017), com boa acidez e ligeiro amargor, apresentando-se com potencial para amadurecimento, não muito longo, podendo ser utilizada na elaboração de vinhos tranquilos. (GIOVANINI, 2005).

O uso de barricas de carvalho para amadurecer o vinho e outras bebidas já é feito há séculos (GOMES-DE-PINA et al., 2000). Segundo Ribéreau Gayon et al. (2006), a prática de fermentar e conservar vinhos brancos em barricas de carvalho teve início no século XX em Borgonha, na França, e, durante os anos 80 , espalhou-se a quase todas as regiões vitivinícolas do mundo. Desse modo, nos últimos anos, países que não pertencem à União Europeia como Austrália, Estados Unidos, Chile, e outros, que não têm regulamentos restritivos na indústria do vinho, estão introduzindo nesse mercado fragmentos de carvalho como alternativa para a maturação tradicional em barricas (PÉREZ-MAGARIÑO et al., 2009; PRIETO et al., 2012; CHIRA; TEISSEDRE, 2013).

O chip de carvalho é um insumo enológico, que representa uma alternativa econômica para atribuir aromas terciários, transferindo rapidamente compostos aromáticos para o vinho, podendo substituir, em parte, o uso dos barris. Este insumo tem variadas características no que diz respeito à sua tostagem e composição, podendo ser deixados no estado natural ou serem aquecidos de modo ligeiro (suave), médio ou forte, mas não devem ter sofrido combustão (PATACO, 2013).

Os fragmentos de carvalho quando incorporados liberam compostos, como baunilha e furfural, promovendo uma maior complexidade ao vinho. Conforme o grau de tostagem da madeira, diferentes aromas irão ser agregados ao vinho, podendo ir da baunilha a aromas mais intensos, como o de café e pão tostado (CHATONNET, 2007).

Neste sentido, este trabalho teve como 0 objetivo do trabalho é avaliar o efeito da utilização de chips de carvalho francês na composição físico-química em vinhos brancos tranquilos com a cv. Viognier na região do Submédio do Vale do São Francisco.

\section{Material e métodos}




\section{Matéria-prima}

A variedade utilizada no estudo foi a cv. Viognier provenientes da Vinícola Santa Maria (Vinhos Rio Sol), localizada em Santa Maria da Boa Vista - PE. A uva foi destinada a Escola do Vinho, localizada no Instituto Federal de Educação Ciência e Tecnologia do Sertão Pernambucano, BR 647- KM 22, Zona Rural- Petrolina - PE. A uva foi recebida em caixas de plástico de $20 \mathrm{Kg}$ de capacidade, onde foram pesadas totalizando $500 \mathrm{Kg}$ de uvas.

\section{Local do experimento}

O experimento foi conduzido nas dependências do Instituto Federal de Educação, Ciência e Tecnologia do Sertão Pernambucano, Campus Petrolina Zona Rural: Os vinhos foram elaborados na Escola do Vinho, as análises clássicas realizadas no laboratório de química deste mesmo setor e as análises cromatográficas na Embrapa Semiárido.

\section{Delineamento experimental}

Conduziu-se o experimento em delineamento inteiramente casualizado em sistema fatorial 4 tratamentos com 3 repetições. 0 experimento consistiu na elaboração de vinhos tranquilos, a partir da variedade Viognier conforme na Tabela 1. Tabela 1: Codificação e caracterização dos tratamentos.

\begin{tabular}{cc}
\hline Tratamentos & \multicolumn{1}{c}{ Caracterização } \\
\hline T0 & Sem adição de chip de carvalho francês \\
T1 & Com adição de $2 \mathrm{~g} \cdot \mathrm{L}^{-1}$ de chip de carvalho francês \\
T3 & Com adição de $4 \mathrm{~g} \cdot \mathrm{L}^{-1}$ de chip de carvalho francês \\
\hline
\end{tabular}

\section{Elaboração do vinho}

O processo de vinificação ocorreu de forma tradicional de vinhos brancos, conforme método tradicional de Peynaud, (1982). Para extração do mosto, as uvas foram submetidas ao processo de desengace e esmagamento de forma manual para assegurar uma maior integridade da baga. Durante o desengace realizou-se a sulfitagem e enzimagem nas concentrações de 50 mg. $\mathrm{L}^{-1}$ para a solução de dióxido de enxofre a $5 \%$ e $2 \mathrm{~mL} \cdot \mathrm{hL}^{-1}$ da enzima Everzym Thermo ${ }^{\circledR}$. Após realizou-se a prensagem de forma direta, isto é, sem que ocorresse maceração, com o auxílio de uma prensa manual.

Realizou-se a debourbage dos mostos na câmara fria a uma temperatura de $(6 \pm 2)^{\circ} \mathrm{C}$, durante 24 horas. Em seguida, iniciou-se o processo de fermentação alcoólica utilizou-se 20 $\mathrm{g} / \mathrm{hL}^{-1}$ da levedura Maurivin PDM $\otimes$. Os garrafões foram submetidos à temperatura de $(16 \pm 2)^{\circ} \mathrm{C}$, na câmara fria, com análise de densidade e controle da temperatura duas vezes ao dia, realizando-se também duas remontagens diárias até o final da fermentação. A adição dos chips de carvalho francês foi sucedida após dois dias do início da fermentação alcoólica, e para isto foram adicionados os chips nas seguintes concentrações: $2 \mathrm{~g} \cdot \mathrm{L}^{-1}$, $3 \mathrm{~g} \cdot \mathrm{L}^{-1}$ e $4 \mathrm{~g} \cdot \mathrm{L}^{-1}$. A retirada dos chips aconteceu após 16 (dezesseis) dias de fermentação mediante análise sensorial. A conclusão da fermentação se deu no 18 (décimo oitavo) dia mediante análise de densidade. Após 12 (doze) dias de fermentação alcoólica determinou-se o final desta etapa com análises das densidades, teores alcoólicos e açúcares redutores.

Com auxílio do frio, os vinhos foram submetidos à temperatura de $(-3 \pm 1)^{\circ} \mathrm{C}$ na câmara fria por cerca de 10 (dez) dias, realizando a estabilização tartárica. Para a estabilidade protéica utilizou-se a dosagem de $80 \mathrm{~g} \cdot \mathrm{hL}^{-1}$ da bentonite MAXIBENT PLUS $\circledast$, para eliminar as proteínas instáveis presentes no vinho. As etapas da vinificação ocorreram conforme a Figura 1. 
Figura 1: Fluxograma de elaboração do vinho.

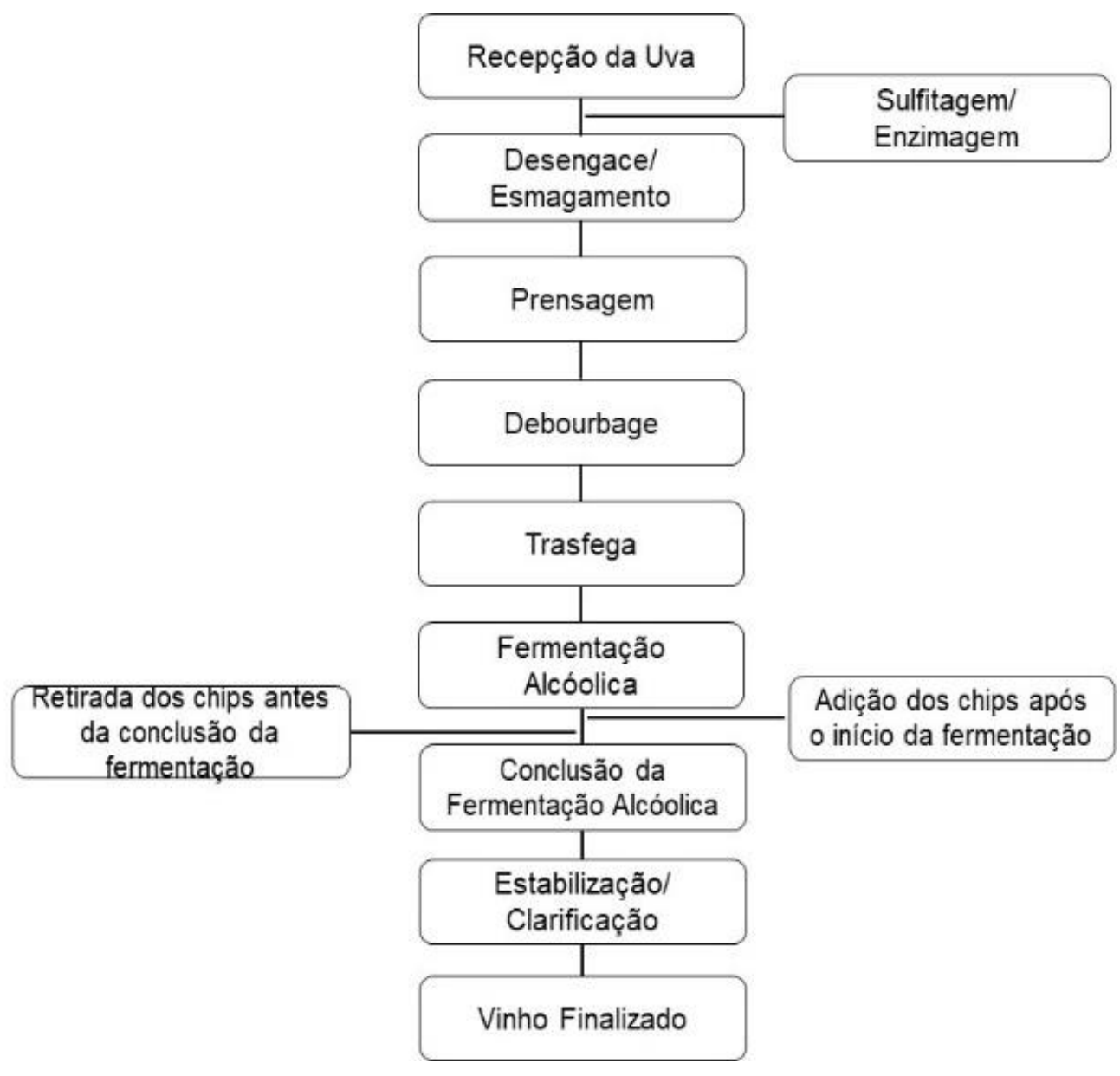

\section{Análises físico-químicas}

As análises físico-químicas realizadas foram: densidade relativa $\left(\mathrm{g} . \mathrm{cm}^{-3}\right)$, teor álcool (\% $\mathrm{v} / \mathrm{v})$, acidez total titulável (g. $\left.\mathrm{L}^{-1}\right)$, acidez volátil bruta $\left(\mathrm{g} \cdot \mathrm{L}^{-1}\right), \mathrm{pH}$, dióxido de enxofre $\left(\mathrm{SO}_{2}\right)$ livre e total (g. $\left.\mathrm{L}^{-1}\right)$, extrato seco total (g. $\left.\mathrm{L}^{-1}\right)$ e extrato seco reduzido (g. $\left.\mathrm{L}^{-1}\right)$ (OAC, 2005). A análise de açúcares redutores (g. $\left.\mathrm{L}^{-1}\right)$ foi realizada através da metodologia de Ribereau-gayon et al. (1980) e a análise colorimétrica, utilizando método do Ministério de Agricultura, Pecuária e abastecimento (BRASIL, 2005).

\section{Análise estatística}

Os resultados obtidos foram submetidos à análise de variância (ANOVA), e comparados pelo teste de Tukey ao nível de 5\% de significância.

\section{Resultados e discussão}

\section{Análises clássicas}

Os resultados das análises físico-química do vinho estão apresentados na Tabela 2 estas foram realizadas em triplicatas. 
Tabela 2: Resultados das análises físico-químicas do vinho a partir da cv. Viognier.

\begin{tabular}{|c|c|c|c|c|}
\hline \multicolumn{2}{|c|}{ PARÂMETROS } & \multicolumn{3}{|c|}{ TRATAMENTOS } \\
\hline & TO & T1 & T2 & T3 \\
\hline $\begin{array}{l}\text { Densidade } \\
\text { relativa }\left(\mathrm{g} \cdot \mathrm{cm}^{-}\right. \\
{ }^{3} \text { ) }\end{array}$ & $0,990^{a}$ & $0,990^{a}$ & $0,990^{a}$ & $0,990^{a}$ \\
\hline $\begin{array}{l}\text { Teor } \\
\text { alcoólico \% }\end{array}$ & $14,0^{\mathrm{a}}$ & $14,0^{a}$ & $14,0^{a}$ & $14,0^{a}$ \\
\hline $\begin{array}{l}\text { Açúcar redutor } \\
\left(\mathrm{g} \cdot \mathrm{L}^{-1}\right)\end{array}$ & $2,03^{a}$ & $1,92^{\mathrm{a}}$ & $1,85^{a}$ & $1,91^{\mathrm{a}}$ \\
\hline $\begin{array}{l}\text { Acidez Total } \\
\text { Titulável (g.L.1) }\end{array}$ & $6,0^{\mathrm{b}}$ & $5,89^{c}$ & $5,87^{a}$ & $5,91^{\mathrm{b}}$ \\
\hline $\begin{array}{l}\text { Acidez Volátil } \\
\left(\text { g. } \text { L }^{-1}\right)\end{array}$ & $0,98^{a}$ & $0,75^{\mathrm{b}}$ & $0,79^{b}$ & $0,79^{b}$ \\
\hline $\mathrm{pH}$ & $3,85^{\mathrm{a}}$ & $3,84^{\mathrm{a}}$ & $3,85^{\mathrm{a}}$ & $3,83^{a}$ \\
\hline $\mathrm{SO}_{2}$ Total (g. $\left.\mathrm{L}^{-1}\right)$ & $0,102^{b}$ & $0,090^{c}$ & $0,120^{a}$ & $0,110^{b}$ \\
\hline $\mathrm{SO}_{2}$ Livre $\left(\mathrm{g} \cdot \mathrm{L}^{-1}\right)$ & $0,020^{\mathrm{b}}$ & $0,020^{\mathrm{b}}$ & $0,040^{a}$ & $0,040^{a}$ \\
\hline $\begin{array}{l}\text { Extrato Seco } \\
\text { Total }\left(\mathrm{g} \cdot \mathrm{L}^{-1}\right)\end{array}$ & $20,09^{a}$ & $20,09^{a}$ & $20,09^{a}$ & $20,09^{a}$ \\
\hline Extrato Seco & & & & $18,99^{a}$ \\
\hline $\begin{array}{l}\text { Reduzido (g.L - } \\
\text { 1) }\end{array}$ & $18,87^{\mathrm{a}}$ & $18,98^{a}$ & $19,05^{\mathrm{a}}$ & \\
\hline
\end{tabular}

*Médias seguidas de mesma letra nas linhas, não diferem significativamente entre si pelo teste de Tukey a $5 \%$ de probabilidade. T0- Sem adição de chip de carvalho francês; T1- Com adição de $2 \mathrm{~g} . \mathrm{L}^{-1}$ de chip de carvalho francês; T2- Com adição de $3 \mathrm{~g} . \mathrm{L}^{-1}$ de chip de carvalho francês; T3Com adição de $4 \mathrm{~g} \cdot \mathrm{L}^{-1}$ de chip de carvalho francês.

Para o aspecto densidade, os vinhos elaborados não apresentam diferenças significativas. Os valores encontrados para os tratamentos (T0, T1, T2 e T3) foram de 0,990 g. $\mathrm{cm}^{-3}$. O resultado encontrado está de acordo com o descrito por Mathias (2018), em que determina que a densidade final do vinho deve ficar entre 0,990 e $0,995 \mathrm{~g} . \mathrm{cm}^{-3}$.

0 teor alcoólico das amostras de vinho elaborados sem e com chip de carvalho para os tratamentos T0, T1, T2 e T3 variaram entre 14,0 e 14,1\%. De acordo com Brasil (2008), o vinho branco fino apresenta teor alcoólico entre $8,6 \%$ (oito inteiros e seis décimos por cento) e 14,0\% (catorze por cento) em volume.

Os resultados encontrados para o aspecto açúcar redutor, mostram que não houve diferença significativa entre os tratamentos realizados T0, T1, T2 e T3. Estes valores indicam que os vinhos elaborados se apresentam secos, pois o açúcar residual se encontra abaixo de $4 \mathrm{~g} / \mathrm{L}^{-1}$ (BRASIL, 2008). Os valores de acidez total para os tratamentos variam entre 5,89 e 6,00 g. L-1 em ácido tartárico, resultado menor do que encontrado por Lima (2010) que foi de 5,58 g.L ${ }^{-1}$, também na cv. Vioginer no Submédio Vale do São Francisco.

Para o parâmetro acidez volátil os valores variaram entre 0,98 e $0,7 \mathrm{~g}, \mathrm{~L}^{-1} \mathrm{em}$ ácido acético para os tratamentos (T0, T1, T2 e T3). Os valores encontrados estão de acordo com o limite estabelecido na legislação brasileira que determina o valor máximo de $1,2 \mathrm{~g}$. $\mathrm{L}^{-1} \mathrm{em}$ ácido acético para acidez volátil (BRASIL, 2018).

Houve diferença significativa no parâmetro acidez volátil entre o tratamento (T0) e os demais (T1, T2 e T3), esta situação pode ser explicada devido ao contato prolongado entre o vinho e oxigênio durante a elaboração no tratamento T0, por conta do seu menor volume. Na presença de quantidades elevadas de oxigênio as bactérias acéticas produzem o aumento da acidez volátil (DELANOE et al, 2003).

Para o aspecto $\mathrm{pH}$ não houve diferença significativa entre os tratamentos. Observando- 
se que os vinhos elaborados apresentaram valores de $\mathrm{pH}$ entre 3,85 e 3,83, resultados estes superiores aos descritos por Tecchio (2007) em diz que os vinhos brasileiros apresentam pH que variam de 3,0 até 3,6 .

$\mathrm{O}$ teor de anidrido sulfuroso $\left(\mathrm{SO}_{2}\right)$ é um constituinte normal do vinho, sendo resultado da adição como agente antimicrobiano e antioxidante para sua conservação (JACKSON, 2008). De acordo com Giovanni \& Manfroi (2009), $\mathrm{O} \mathrm{SO}_{2}$ pode se apresentar de forma livre (molecular) ou combinada, a soma destas gera o $\mathrm{SO}_{2}$ total, cujo teor máximo permitido pela legislação brasileira é de $350 \mathrm{mg} \cdot \mathrm{L}^{-1} \mathrm{~A}$ concentração de $\mathrm{SO}_{2}$ total nos vinhos elaborados variaram entre 0,090 e 0,120 g.L - $^{-1}$.

Para o parâmetro extrato seco reduzido não houve diferença significativa. Observandose que os vinhos elaborados para os tratamentos (T0, T1, T2 e T3) apresentaram valores entre 18,87 e $19,05 \mathrm{~g} . \mathrm{L}^{-1}$, os resultados encontram-se dentro do estabelecido na legislação brasileira em que determina que o valore mínimo de extrato seco reduzido é de $16 \mathrm{~g} . \mathrm{L}^{-1}$ para vinhos brancos (BRASIL,2018). A legislação brasileira não estabelece limites para extrato seco total dos vinhos.

\section{Análise de cor por espectrofotometria}

Os resultados das análises físico-química do vinho estão apresentados na Tabela 3 estas foram realizadas em triplicatas.

Tabela 3: Resultados dos parâmetros de cor por espectrofotometria do vinho

\begin{tabular}{lcccc}
\hline & PARÂMETROS & \multicolumn{3}{c}{ TRATAMENTOS } \\
\hline & TO & T1 & T2 & T3 \\
\hline $\mathrm{L}(\%)$ & $50,41^{\mathrm{a}}$ & $49,93^{\mathrm{ab}}$ & $49,97^{\mathrm{ab}}$ & $49,73^{\mathrm{b}}$ \\
$\mathrm{a}^{*}$ & $0,97^{\mathrm{d}}$ & $1,45^{\mathrm{c}}$ & $1,67^{\mathrm{b}}$ & $1,78^{\mathrm{a}}$ \\
$\mathrm{b}^{*}$ & $10,48^{\mathrm{b}}$ & $10,65^{\mathrm{a}}$ & $10,25^{\mathrm{d}}$ & $10,37^{\mathrm{c}}$ \\
$\mathrm{C}$ & $31,59^{\mathrm{b}}$ & $32,06^{\mathrm{a}}$ & $31,18^{\mathrm{c}}$ & $31,57^{\mathrm{b}}$ \\
$\mathrm{h}$ & $84,59^{\mathrm{a}}$ & $82,21^{\mathrm{b}}$ & $80,73^{\mathrm{c}}$ & $80,20^{\mathrm{d}}$ \\
(graus) & & & & \\
\hline
\end{tabular}

$\mathrm{L}^{*}$ : luminosidade; $\mathrm{a}^{*}$ e $\mathrm{b}^{*}$ : coordenadas de cor CIELAB; $\mathrm{C}^{*}$ : saturação; $\mathrm{h}^{*}$ : tonalidade: Médias seguidas da mesma letra nas linhas, não diferem significativamente entre si pelo teste de Tukey a $5 \%$ de probabilidade. T0- Sem adição de chip de carvalho francês; T1- Com adição de $2 \mathrm{~g} \cdot \mathrm{L}^{-1}$ de chip de carvalho francês; T2- Com adição de 3g. $\mathrm{L}^{-1}$ de chip de carvalho francês; T3- Com adição de $4 \mathrm{~g} \cdot \mathrm{L}^{-1}$ de chip de carvalho francês.

Foram confirmadas diferenças significativas entre os tratamentos realizados nesse estudo, para os parâmetros colorimétricos $L, a^{*}, b^{*}, C$ e $h^{*}$, que reconhece a diferença dos vinhos tranquilos em uso das diferentes quantidades de chip de carvalho francês de média tostagem. Para o parâmetro luminosidade $\left(\mathrm{L}^{*}\right)$ os resultados mostram diferenças significativas entre os tratamentos (T0) com 50,41\% apresentando maior índice, indicando maior clareza, consequentemente maior luminosidade, e o tratamento (T3) com 49,73\% tendo menor índice, indicando menor clareza e luminosidade. Este fato infere que o vinho (T0) exibe uma maior transparência em comparação ao vinho (T3). Os tratamentos (T1 e T2) não demostram diferenças significativas, entretanto existe uma relação com os demais tratamentos.

Para a coordenada a* (vermelho/verde) os vinhos (T0, T1, T2 e T3) apontaram componentes de cor vermelha, tendo o maior valor em T3 $(1,78)$, indicando que com a utilização de uma maior concentração de chip de carvalho, ocorra uma maior concentração de componentes de cor vermelha. Na coordenada b* (amarelo/azul) os tratamentos (T0, T1, T2 e T3) apresentaram diferenças significativas entre si, com valores entre 10,65 e 10,25, contudo ambos os tratamentos apontam componentes de cor amarelo. Esta característica é esperada em vinhos brancos que em tiveram a presença de carvalho em algum momento do processo de 
elaboração (LAUREANO et al., 1998). Para a coordenada C* (saturação de cor) houve diferença significativa entre os tratamentos ( $\mathrm{T} 1$ e T2) com valores de 32,06 e 31,18 respectivamente. Os demais tratamentos não obtiveram diferenças significativas.

Para o ângulo $\mathrm{h}^{*}$ (tonalidade) os tratamentos alcançaram resultados com diferenças significativas, onde o vinho (T0) sucedeu o maior valor 84,59 em comparativo com os demais tratamentos (T1, T2 e T3) 82,$21 ; 80,73 ; 80,20$, respectivamente. É evidente que o tipo e concentração utilizado no estudo não teve influência nos resultados. Pois, o valor aproximado para este fator foi encontrado por Lima (2010) em um vinho da cv. Viognier cultivada no Vale do Submédio São Francisco, com o valor de 85, $9^{\circ}$.

\section{Conclusão}

Os vinhos elaborados a partir da incorporação de chip de carvalho francês de média tostagem com a cultivar Viognier apresentaram características físico-químicas dentro do permitido pela legislação vigente, demonstrando assim a possibilidade de sua utilização dos fragmentos industrial para elaboração de vinhos brancos.

0 chip de carvalho de média tostagem favorece a presença da coloração amarela característica esperada em vinhos brancos com seu emprego.

Tendo em vista os aspectos observados, conclui-se, as três concentrações de chips de carvalho francês de média tostagem são recomendados para elaboração de vinho branco com a cultivar Viognier, pois garante que os parâmetros físico-químicos permanecem dentro dos padrões estabelecidos para a elaboração da bebida.

Tornam-se necessários estudos complementares com o intuito de explorar o efeito do uso de chips em vinhos, tendo-se em vista que a utilização de chip é uma alternativa mais econômica e que representa extensos benefícios na elaboração de vinhos brancos sem comprometer a qualidade final do produto, podendo, dessa forma, ampliar a diversidade de produtos vitivinicolas no mercado mundial.

\section{REFERÊNCIAS}

AOAC. Official methods of analysis of the Association Analytical Chemists. 18.ed. Gaithersburg, Maryland, 2005.

BRASIL, Ministério da Agricultura e do abastecimento. Complementação dos padrões e identidade e qualidade do vinho e dos derivados da uva e do vinho. 2004. Disponível em < http://www.planalto.gov.br/ccivil_03/_ato2004-2006/2004/lei/l10.970.htm>Acesso

em maio.2019.

DELANOE, D.; MAILLARD, G.; MAISINDIEU, D. El Vino: del análisis a la elaboración. 5. ed. Editorial Acribia: Zaragoga, Espanha. 2003. p. 10-16.

FRAIGE K (2012) Estudo comparativo do perfil metabolômico e proteômico de uvas (vitis vinifera) durante o processo de maturação utilizando ferramentas bioanalíticas. Tese apresentada ao Instituto de Quimíca de São Carlos da Universidade de São Paulo para obtenção do título de doutor em ciências. São Carlos. SP, 2012.

IBRAVIN, INSTITUTO BRASILEIRO DO VINHO. Bento Gonçalves. Disponível em <http://www.ibravin.org.br/downloads/1377636150> Acesso em abril 2018.

IBRAVIN, INSTITUTO BRASILEIRO DO VINHO. Bento Gonçalves. Disponível em < 

maio.2019.

LIMA, L.L.A. Caracterização e estabilização dos vinhos elaborados no Vale do Submédio São Francisco. Tese para obtenção de doutorado em nutrição da universidade de Pernambuco. Recife-PE. $2010 . \quad$ p.108. Disponível em< https://repositorio.ufpe.br/bitstream/123456789/8137/1/arquivo3062_1.pdf>Acesso em maio.2019.

LOPES, P. R. C., et al. Produção integrada de uva para vinho - PI-UV. 2008. Disponível em <http:/ / www.bdpa.cnptia.embrapa.br/consulta/busca?b=ad\&id=160980\&biblioteca=vazio\&tbu sca $=160980 \& q F a c e t s=160980 \&$ sort $=\&$ tpaginacao $=$ t\&paginaAtual=1 $>$ Acesso em outubro 2019 . MAPA, Ministério da Agricultura e Pecuária. Complementação dos padrões de identidade e qualidade do vinho. 1998. Disponível em < http://www.uvibra.com.br/legislacao_portaria229.htm>Acesso em maio.2019.

MATHIAS; T.R.S. Tecnologia do vinho. Disponível em: < http://semanadaquimica.org/wpcontent/uploads/2018/04/Curso-bebidas-fermentadas-Vinho.pdf> Acesso em jul.2019.

OIV. International Organizatios of vine and wine, State of the vitiviniculture world Market. Disponível em: <http://www.oiv.int/public/medias/6779/state-of-the-vitiniculture-worldmarket-oiv-2019-congress-pr.pdf > acesso em Jul. 2019.

PEREIRA, G. E.; GUERRA, C. C.; MANFROI, L. Viticultura e enologia. Ln: SOARES, J. M; LEÃO, P. C. de $S$ (Eds.) A vitivinicultura no Semiárido brasileiro. Petrolina, PE: Embrapa Semiárido, 2009, p. 677-724. requisitos para conclusão do Curso Superior de Tecnologia em Viticultura e Enologia. Bento Gonçalves.2007. p.47. Disponível em< https://docplayer.com.br/20591177Caracteristicas-fisico-quimicas-e-sensoriais-do-vinho-bordo-de-flores-da-cunha.html>Acesso em maio.2019.

RIZZON, L.,A.; MIELE A. (1986). Extrato Seco Total de Vinhos Brasileiros: Comparação de Métodos Analíticos. Disponível em < http://www.scielo.br/pdf/cr/v26n2/a22v26n2.pdf > Acesso em maio.2019.

SANTOS, A.O.; ROLIM, G. S.; HERNANDES, J.L.; PEDRO JÚNIOR, M.J. A maturação fisiológica da videira vinífera em Sâo Paulo: Comentários sobre as safras de verão e de inverno na média altitude paulista. 2009. Artigo em Hypertexto. Disponível em: < http://www.iac.sp.gov.br/imagem_informacoestecnologicas/53.pdf>.Acesso em jul.2019.

SILVA, F. G Potencialidade de variedades viníferas para produção de vinhos finos na Microrregião de Garanhuns-PE. Dissertação de Mestrado, da Universidade Federal Rural de Pernambuco, PPGAMGP, Recife-PE, 2017.

SILVA,. I.S; NOGUEIRA,. E.T; et al. Influência do uso de chip de carvalho frânces na composição físico-química e colorimétrica de vinho tinto 'syrah'. IV Encontro Nacional da 2018 Disponível em https://www.alice.cnptia.embrapa.br/alice/bitstream/doc/1103648/1/AC4proceedingsIVENA G98023.pdf> Acesso em jul. 2019.

SOARES J. M., LEÃO P. C. S. A vitivinicultura no Semiárido Brasileiro. Brasília, DF: Empraba Informação Tecnológica; Petrolina: Embrapa Semiárido, 2009. 
SOUSA, S. I. (2005) Espumante: o prazer é todo seu. Marco Zero, São Paulo, 165 p.

TECCHIO. Características Físico-Químicas e Sensoriais do Vinho Bordô de Flores da Cunha. Monografia apresentada como um dos

TOGORES, Hidalgo. Tratado de Enología. Volume I e II. $1^{\text {a }}$ ed. Espanha (Madrid): Mundi Prensa, 2003.

VARGAS, J., F., Influência de chips de carvalho em diferentes épocas de aplicação e tempo de relação sobre vinhos Chardonnay da região da Campanha Gaúcha. Obtenção do título de Bacharel em Enologia. Dom Pedrito, RS, 2017. 